\title{
Effects of androgens and estrogens on sirtuin 1 gene expression in human aortic endothelial cells
}

Takafumi Tsuchiya, MD, PhD, Ayano Takei, BS, Kyoko Tsujikado, BS, Toshibiko Inukai, MD, PhD.

\begin{abstract}
الأهداف : دراسة تأثير الأندروجينات والاستروجين على تعبير السورتوين 1 البانية

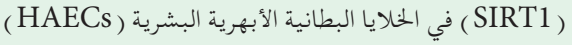

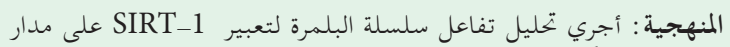

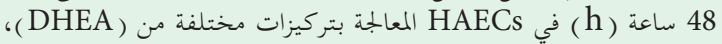

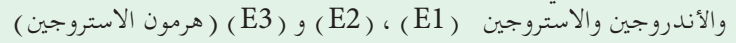

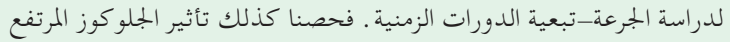

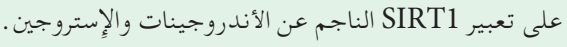

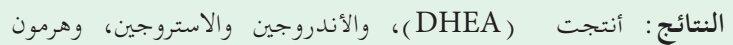

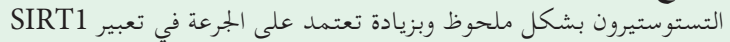

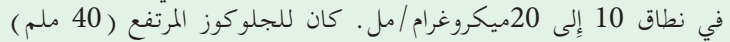

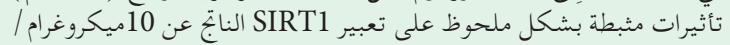

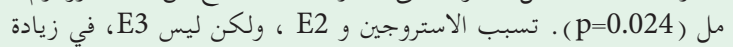

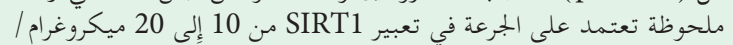

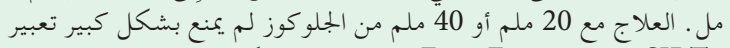

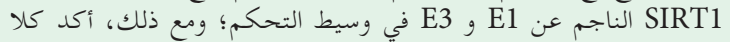

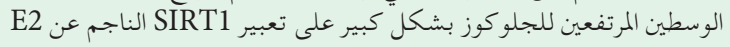
في وسط التحكم ( P=0.007، p=0.005)، على التوالي.

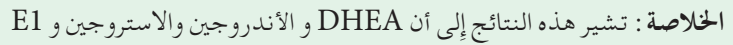

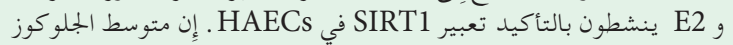

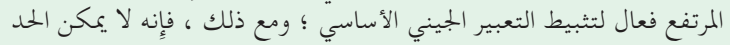

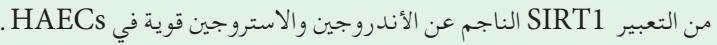

Objectives: To investigate the effect of androgens and estrogens on surtuin 1 (SIRT1) expression in human aortic endothelial cells (HAECs).

Methods: Real-time polymerase chain reaction analysis of SIRT-1 expression over 48 hours (h) was performed in HAECs treated with various concentrations of dehydroepiandrostendione(DHEA), androstenedione and testosterone (androgens), estrone (E1), estradiol (E2), and estriol (E3) (estrogens) to investigate the dose-dependency of time courses. The influence of high glucose on SIRT1 expression induced by the androgens and estrogens was also examined.

Results: androstenedione, and testosterone remarkably produced a dose-dependent increase in SIRT1 expression in the range of 10 to $20 \mu \mathrm{g} / \mathrm{ml}$. High glucose $(40 \mathrm{mM})$ medium had significantly inhibitory effects on $10 \mu \mathrm{g} / \mathrm{ml}$ DHEA-induced SIRT1 expression $(p=0.024)$. Estrone and E2, but not E3, caused a marked dose-dependent increase in SIRT1 expression from 10 to $20 \mu \mathrm{g} / \mathrm{ml}$. Treatment with $20 \mathrm{mM}$ or 40 $\mathrm{mM}$ glucose medium did not significantly inhibit E1and E3-induced SIRT1 expression in control medium; however, both high glucose mediums significantly emphasized E2-induced SIRT1 expression in control medium ( $p=0.007, p=0.005)$.

Conclusion: These results suggest that DHEA, androstenedione, testosterone, E1, and E2 definitely activate SIRT1 expression in HAECs. A high glucose medium is potent to inhibit the basal gene expression; however, it could not reduce powerful androgen- and estrogen-induced SIRT1 expression in HAECs.

Keywords: androgen, estrogen, sirtuin 1, HAECs, high glucose

Saudi Med J 2020; Vol. 41 (4): 361-368

doi: $10.15537 /$ smj.2020.4.25006

From the Department of Internal Medicine (Tsuchiya), from the Department of Joint Research Center (Takei, Tsujikado), Dokkyo Medical University, Saitama Medical Center, Koshigaya; and from the Department of Diabetes and Endocrinology (Inukai), Seibu General Hospital, Saitama, Japan.

Received 13th October 2019. Accepted 9th February 2020.

Address correspondence and reprint request to: Dr. Toshihiko Inukai, Director, Seibu General Hospital, Saitama, Japan.E-mail:t-inukai@seibu-hospital.or.jp ORCHID ID: https://orcid.org/0000-0002-8539-1615 
$\mathrm{D}$ elineating hormonal signaling changes that occur across a lifespan and searching interventions may improve the quality of life (QOL) of elderly people and activate longevity. ${ }^{1}$ Surtuin 1 (SIRT1) is a key gene associated with longevity. Surtuins regulate steroid hormone signaling through a variety of molecular mechanisms and modulate pathways that modify steroid hormone receptors through phosphorylation. ${ }^{2}$

Polyphenols and $\omega$-3-line polyunsaturated fatty acids (PUFAs) contribute to anti-aging and are also closely associated with activation of SIRT1 expression in some cultured cells. ${ }^{3}$ Various hormones affect SIRT1 expression in these cells. Neurosteroids play an important role in cognitive function and QOL, and hormones such as androgens and estrogens are especially critical for prevention of atherosclerosis and ageing and promotion of longevity through activation of surtuins. ${ }^{4-7}$ Estrogen- and testosterone-dependent actions play a vital role in mitochondrial processes implicated in aging. ${ }^{8}$ Beneficial effects of dehydroepiandrostendione (DHEA), an androgen, as an anti-aging steroid have been shown in vitro and in vivo, including the stimulation of immunity and then the suppression of diabetes, atherosclerosis, dementia, obesity, and osteoporosis activities. ${ }^{9}$ Estrogens may also modulate cardiovascular health through expression of SIRT1, possibly in the AKT and ERK signal pathways. ${ }^{10}$ The beneficial effects of estradiol appear to occur from its antilipofuscin, antioxidant and anti-lipid peroxidation properties and to imply an overall anti-aging action. ${ }^{11}$ Selective targeting of estrogen receptor $\alpha(\mathrm{ER} \alpha)$ with an agonist potentiates chemotherapy for treatment of ovarian cancer, and suppression of SIRT1 may accelerate this therapeutic effect. ${ }^{12}$

A high glucose medium can inhibit the effect of many different agents. Testosterone regulates $B$-cell mass, at least in part, by androgen receptor (AR) activation in $B$-cells of male rats, and the $B$-cell AR is degraded under hyperglycemic conditions. ${ }^{13}$ In contrast, Chakrabarti et $\mathrm{al}^{14}$ found that control of oxidative stress by adenosine monophosphate-activated protein kinase (AMPK) activation recovers normal estrogen responses, even in the presence of hyperglycemia. Furthermore, at high glucose, kaempferol targets estrogen-related receptor $\alpha$ and suppresses angiogenesis of human aortic endothelial cells (HAECs). ${ }^{15}$

Disclosure. This study was funded by the Department of Internal Medicine, Dokkyo Medical University Saitama Medical Center, Koshigaya, Japan.
Taken together, it has been known the sex hormones are potential to activate the gene associated with longevity and high glucose medium could abolish the hormones' action; however, it is unknown regarding the topic in HAECs as human cells. In order to elucidate these prior unanswered questions, we examined the effects of androgens and estrogens on SIRT1 expression in HAECs, and we investigate whether a high glucose level can affect hormone-induced SIRT1 expression.

Methods. This cross sectional study was conducted at the Joint Laboratory Office (JLO), Dokkyo Medical University Saitama Medical Center, Saitama, Japan from April 2017 to March 2019. The present medical research does not involve human subjects and was approved by our institutional ethical review board, and it is not previously published in partial or full in the website or printed journal in other language than English.

Cell culture. Human aortic endothelial cells were gained from American Type Culture Collection (ATCC Manassas, VA, USA) and cultured in Medium200 containing $10 \%$ large vessel endothelial supplement (LVES) (Gibco, Grand Island, NY, USA) at $37^{\circ} \mathrm{C}$ in a 5\% CO incubator (Thermo Fisher Scientific, Carlsbad, CA, USA), as similar as methods shown in our previous report. $^{3}$ For researches of SIRT1 expression, cells were seeded $\left(10^{5}\right.$ cell $\left./ \mathrm{ml}\right)$ and cultured in medium with 10\% LVES for 24 hours (h), with addition of vehicle (dimethyl sulfoxide [DMSO]), DHEA (Wako Pure Chemical Industries, Japan), androstenedione, testosterone, estrone, estradiol or estriol (Sigma-Aldrich, St. Louis, MO, USA). Cell culture was followed using normalization to DMSO-treated cells.

Assessment of cell viability. After each treatment, the numbers of total and live cells were calculated with trypan blue staining. Cell viability was estimated by counting the ratio of live cells using trypan blue exclusion. ${ }^{16}$

Quantitative real-time polymerase chain reaction (PCR) analysis. Total ribonucleic acid (RNA) was extracted from HAECs using an RNA assay Mini Kit (Qiagen, Hilden, Germany), as similar as methods shown in our previous report. ${ }^{3}$ The complementary deoxyribonucleic acid was compounded using a SuperScript3 first-strand synthesis system for reverse transcription polymerase chain reaction (RT-PCR) (Invitrogen Life Technologies, Carlsbad, CA, USA). The RT-PCR was conducted in duplicate using FastStart SYBR Green Master Super Mix (ROX) (Roche Applied Science, Basel, Switzerland) in a 7500 Fast RT-PCR System (Applied Biosystems, Foster City, CA, USA). 
Data were normalized to $ß$-actin and are presented as the SIRT1/ $\beta$-actin densitometric ratio.

Surtuin 1 expression in HAECs was examined using real-time PCR analysis. Dehydroepiandrostendione, androstenedione and testosterone (androgens), and estrone (E1), estradiol (E2), and estriol (E3) (estrogens) were added to HAECs at various doses, and time courses of SIRT1 expression were determined. The effects of glucose (5 to $40 \mathrm{mM}$ ) on hormone-induced SIRT 1 gene expression were also examined.

Statistical analysis. Data are presented as the mean \pm standard deviation (SD). Statistical analyses of means were performed by analysis of variance (ANOVA), followed by a Student t-test, using the Statistical Package for Social Sciences (Japan IBM, Tokyo, Japan). Comparisons among multiple groups were evaluated by Bonferroni test if the ANOVA was significant. A $p<0.05$ was considered statistically significant.

Results. Time course of SIRT1 expression. Surtuin 1 expression at 6,12, 24 and $48 \mathrm{~h}$ after treatment of HAECs was examined using quantitative RT-PCR analysis, with each experiment repeated 3 or 6 times. Surtuin 1 expression was not observed at 6 and $12 \mathrm{~h}$, but was detected at 24 and $48 \mathrm{~h}$ (data not shown). Therefore, SIRT1 expression at $24 \mathrm{~h}$ was used in all studies shown below.

Dose-dependence of SIRT1 expression. In treatment with androgens, SIRT1 expression was markedly and dose-dependently elevated by DHEA from 0 to $40 \mu \mathrm{g} /$ $\mathrm{ml}$ (Figure 1A), especially both $10 \mu \mathrm{g} / \mathrm{ml}(p=0.013)$ and $20 \mu \mathrm{g} / \mathrm{ml}$ DHEA $(p=0.027)$ were significant elevated compared with control values. Surtuin 1 expression was also significantly affected by androstenedione from 10 to $20 \mu \mathrm{g} / \mathrm{ml}$ (Figure $1 \mathrm{~B}$ ), which showed that both $10 \mu \mathrm{g} / \mathrm{ml}$ androstenedione $(p=0.020)$ and $20 \mu \mathrm{g} / \mathrm{ml}$ androstenedione $(p=0.019)$ were significant stimulated on the gene expression. Furthermore, SIRT1 expression was dose-dependently elevated by testosterone in both $10 \mu \mathrm{g} / \mathrm{ml}(p=0.003)$ and $20 \mu \mathrm{g} / \mathrm{ml}(p=0.0003)$ (Figure 1C). With estrogens, SIRT1 expression was significantly elevated dose-dependently by E1 from $10(p=0.037)$ and $20 \mu \mathrm{g} / \mathrm{ml}(p=0.015)$ (Figure 2), with almost 4-times higher expression at $20 \mu \mathrm{g} / \mathrm{ml} \mathrm{E1}$ compared with control medium. Similarly, E2 from 10 $(p=0.011)$ to $20 \mu \mathrm{g} / \mathrm{ml}(p=0.010)$ dose-dependently increased SIRT1 expression with a significant alteration (Figure 2); however, E3 from $10(p=0.314)$ to $20 \mu \mathrm{g} / \mathrm{ml}$ $(p=0.261)$ had no significant effect on SIRT1 expression (Figure 2).

Influence of high glucose on hormone-induced SIRT1 expression. Both $10 \mathrm{mM}(p=0.0007)$ and $20 \mathrm{mM}$
( $p=0.0002)$ glucose significantly inhibited hormoneinduced SIRT1 expression compared to control $5 \mathrm{mM}$ glucose, and then $20 \mathrm{mM}$ glucose tended to show greater inhibition than $10 \mathrm{mM}$ glucose (Figure 3). With androgens, $40 \mathrm{mM}$ glucose significantly inhibited $10 \mu \mathrm{g} / \mathrm{ml}$ DHEA-stimulated SIRT1 expression compared to $5 \mathrm{mM}$ glucose did $(p=0.024)$ (Figure 3). Both $20 \mathrm{mM}(p=0.221)$ and $40 \mathrm{mM}$ glucose $(p=0.106)$ tended to inhibit SIRT1 expression induced by $10 \mu \mathrm{g} / \mathrm{ml}$ androstenedione with $5 \mathrm{mM}$ glucose (control), but the differences were not statistically significant (Figure 3). As well as data from androstenedione, both $20 \mathrm{mM}$ $(p=0.205)$ and $40 \mathrm{mM}$ glucose $(p=0.098)$ tended to suppress $10 \mu \mathrm{g} / \mathrm{ml}$ testosterone -induced gene expression with $5 \mathrm{mM}$ glucose (control); however, the differences did not lead to statistical significance (Figure 3).

For estrogens, $20 \mathrm{mM}(p=0.401)$ and $40 \mathrm{mM}$ glucose $(p=0.291)$ did not significantly inhibit SIRT1 expression stimulated by $10 \mu \mathrm{g} / \mathrm{ml}$ E1with $5 \mathrm{mM}$ glucose (control) (Figure 4). Similarly, $20 \mathrm{mM}(p=0.465)$ and $40 \mathrm{mM}$ glucose $(p=0.302)$ did not show any significant inhibition on SIRT1 expression stimulated by $10 \mu \mathrm{g} / \mathrm{ml}$ E3 with $5 \mathrm{mM}$ glucose (control) (Figure 4). Interestingly, $20 \mathrm{mM}(p=0.007)$ and $40 \mathrm{mM}$ glucose $(p=0.005)$ significantly emphasized SIRT1 expression induced by $10 \mu \mathrm{g} / \mathrm{ml} \mathrm{E2}$ with $5 \mathrm{mM}$ glucose (control) (Figure 4).

Discussion. The new and important aspects of the present study are that we successfully verified the activated effect of sex hormones such as DHEA, androstenedione and testosterone, E1, E2 on SIRT1 gene expression in HAECs, and then the inhibitory effect of high glucose medium on the gene expression, but not the inhibition of high glucose medium on those hormones-induced gene expression. We provided the novel information as regards the effect of sex hormones on SIRT1 gene expression in human culture cells such as HAECs through the present study, in addition to those effects already known in animal culture cells.

Decline of sex hormones is involved in the aging process and age-related diseases such as sarcopenia, fall, osteoporosis, cognitive decline, mood disorders, cardiovascular health, and sexual activity. ${ }^{17}$ Therefore, the actions of sex hormones on SIRT1 activity is of interest as a surrogate marker for longevity. In this study, we also investigated the influence of high glucose on these actions.

We found that the androgens DHEA and testosterone, but not androstenedione, augmented SIRT1 expression in a dose-dependent manner. These results are reasonable because DHEA and testosterone are more powerful bioactive hormones 


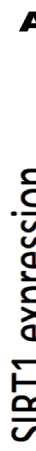

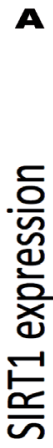

A
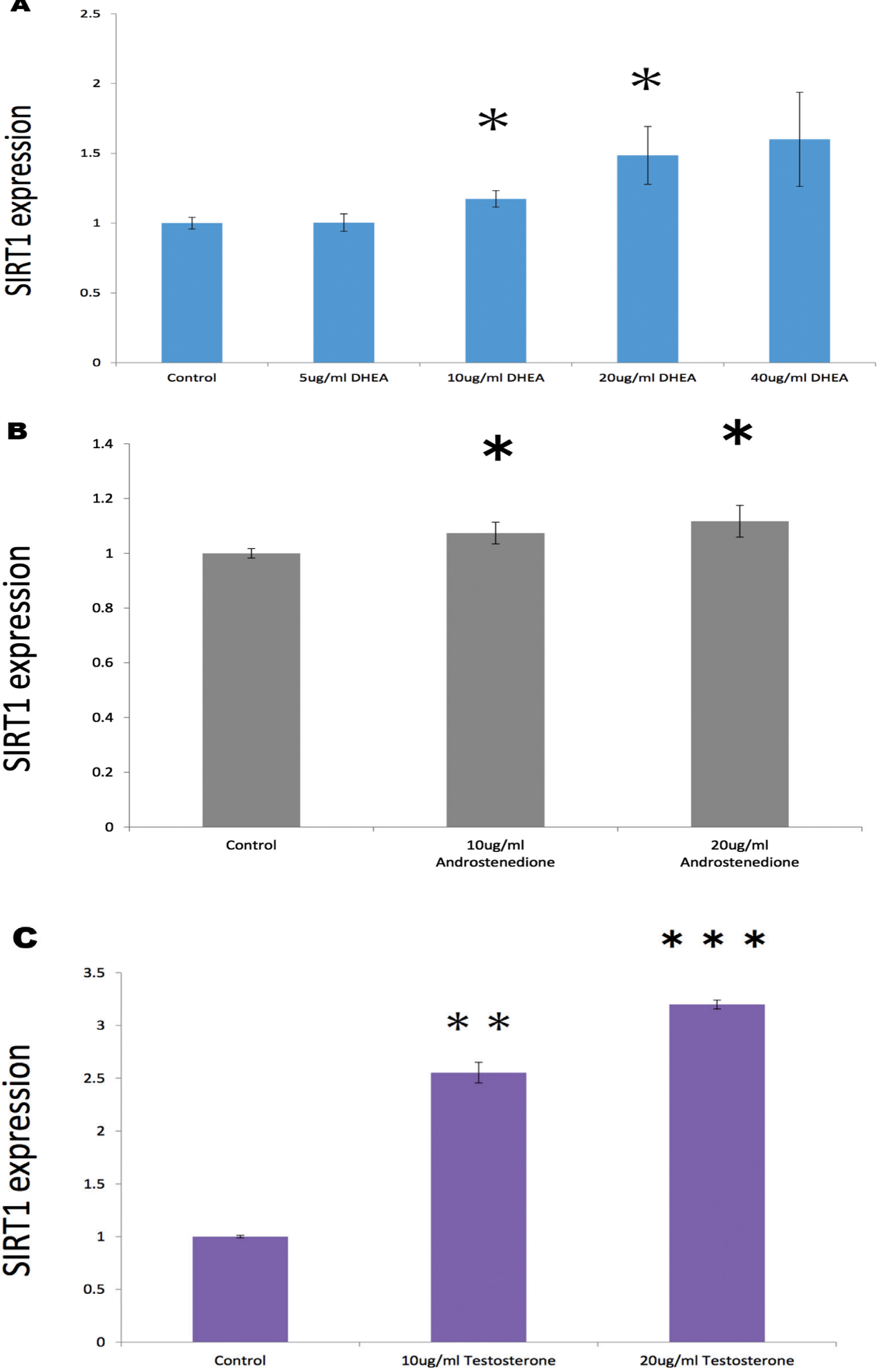

Figure 1 - Effects of A) dehydroepiandrosterone (DHEA) $(0-40 \mu \mathrm{g} / \mathrm{ml}), \mathbf{B})$ androstenedione $(0-20 \mu \mathrm{g} / \mathrm{ml})$, and C) testosterone $(0-20 \mu \mathrm{g} / \mathrm{ml})$ on surtuin 1 (SIRT1) expression in human aortic endothelial cells at 24 hours after the start of treatment. Data are shown as the mean \pm SD of the SIRT1/ßactin densitometric ratio with each agent relative to that with control medium determined in real-time polymerase chain reaction analysis in 2 separate experiments. ${ }^{*} p<0.05,{ }^{* *} p<0.01,{ }^{* * *} p<0.001$ versus control. 


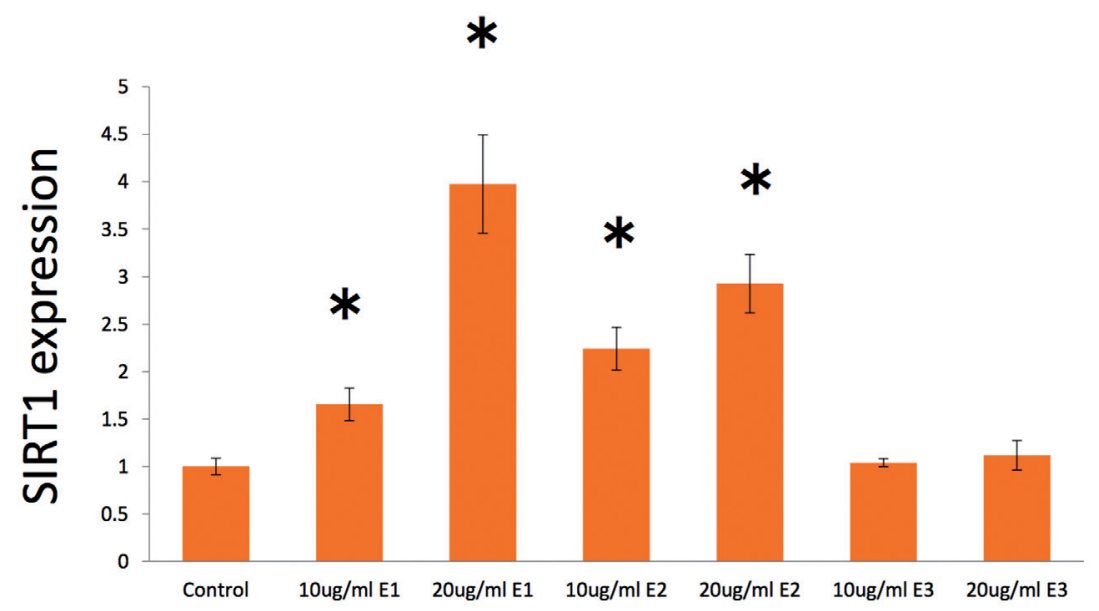

Figure 2 - Effects of estrone, estradiol, and estriol (all 0-20 $\mu \mathrm{g} / \mathrm{ml}$ ) on surtuin 1 (SIRT1) expression in human aortic endothelial cells at 24 hours after the start of treatment. Data are shown as the mean \pm SD of the SIRT1/ßactin densitometric ratio with each agent relative to that with control medium determined in real-time polymerase chain reaction analysis in 2 separate experiments. ${ }^{*} p<0.05$ versus control.

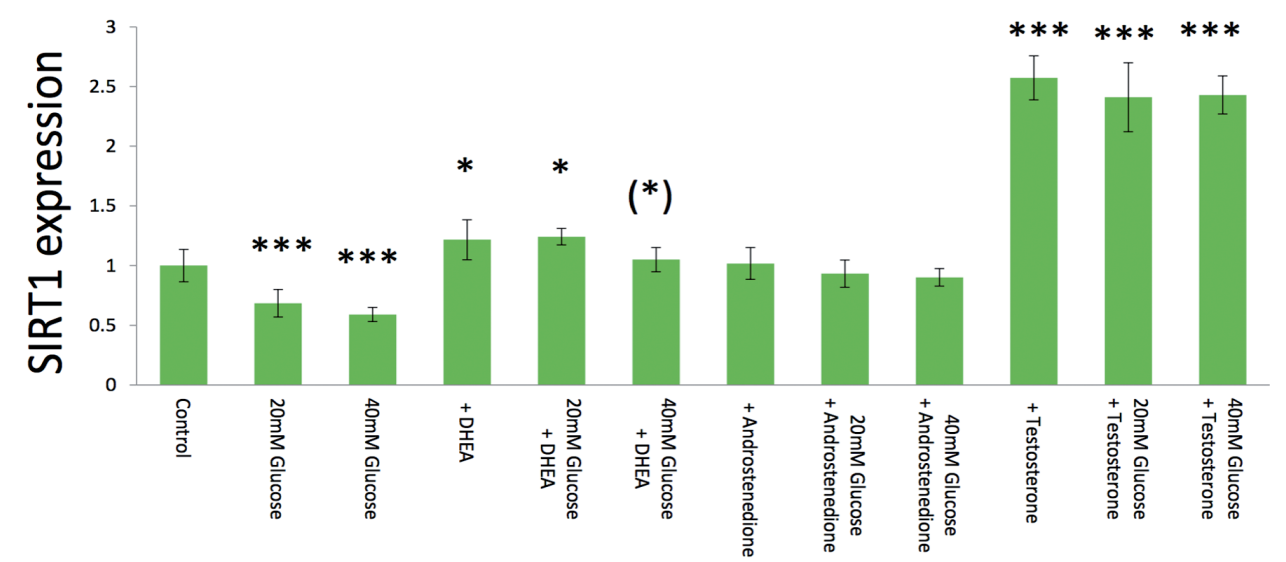

Figure 3 - Glucose (20-40 mM) inhibition of basal, and dehydroepiandrosterone (DHEA), androstenedione-, and testosterone-induced surtuin 1 (SIRT1) expression in real-time polymerase chain reaction analysis. Data are shown as the mean \pm SD of the SIRT1/ $\beta$-actin densitometric ratio with each agent to that from control medium in 2 separate experiments. ${ }^{*} p<0.05,{ }^{* * *} p<0.001$ versus (vs) control, and $\left(^{*}\right) p<0.05$ vs. same agent-induced control medium

than androstenedione. A direct functional link with the $\mathrm{AR}$ is a critical determinant of progression of human prostate cancer and the sirtuins. ${ }^{5}$ Testosterone and SIRT have a critical role in prevention of vascular and neuronal aging. ${ }^{18}$ Based on our results, this effect of testosterone may be evoked through activation of the SIRT gene. Dehydroepiandrosterone inhibits primary rat Leydig cell proliferation by decreasing the cyclin mRNA level, and improves cells viability by modulating the permeability of the mitochondrial membrane and succinate dehydrogenase activity. ${ }^{19}$ These results might be due to the effect of DHEA on activation of
SIRT expression in HAECs, as shown in the current study. Dehydroepiandrostendione prevents linoleic acidinduced endothelial senescence by restoring autophagy and autophagic flux through JNK activation, and the effect of DHEA on longevity might at least partly be associated with this mechanism in HAECs. ${ }^{20}$

We did not find an effect of androstenedione on SIRT1 expression, which is consistent with a report showing that androstenedione had no effect on development of bones, including sternebrae, and soft tissues. ${ }^{21}$ This suggests that androstenedione, a metabolite of the androgen pathway, might have 


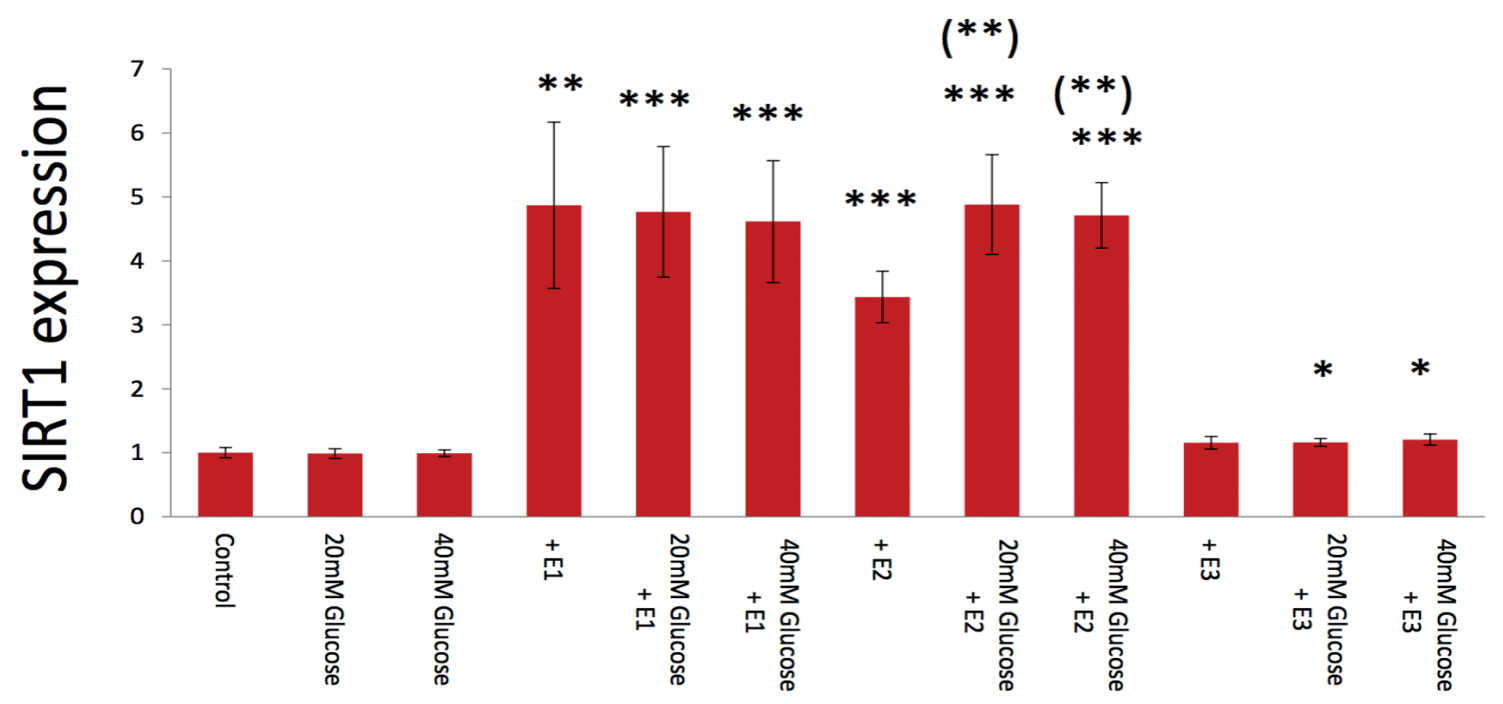

Figure 4 - Glucose (20-40 mM) inhibition of E1-, E2-, and E3-induced SIRT1 expression in real-time PCR analysis. Data are shown as the mean $\pm S D$ of the $S I R T 1 / \beta$-actin densitometric ratio with each agent to that from control medium in 2 separate experiments. ${ }^{*} p<0.05,{ }^{* *} p<0.01,{ }^{* * *} p<0.001$ versus (vs) control, and $\left.{ }^{* *}\right) p<0.01$ vs. same agent-induced control medium.

weak bioactivity in cultured cells. Testosterone and SIRT1 have critical roles in prevention of vascular and neuronal aging, and the present results suggest that this may be partly due to testosterone activation of SIRT1 expression. ${ }^{18}$ Furthermore, testosterone upregulates the anti-aging klotho gene together with AR expression in the kidney, which supports our results despite the difference in the longevity gene. ${ }^{22}$ Novel metabolic pathways linked to male lifespan extension show that male-specific metabolomic responses to $17 \alpha-E 2$ depend on production of testicular hormones in adult life. ${ }^{23}$ This report and the present study show that testosterone plays an important role in longevity.

We also examined the effects of estrogens on SIRT expression. We found effects of E1 and E2, but not E3, on SIRT1 expression. Estrogens protect against ischemic stroke through prevention of neuron death via the SIRT1-dependent AMPK pathway, and this supports our results showing that E1 and E2 activate SIRT1 expression. ${ }^{24}$ Transvaginal E3 potentially offers a suitable physiologic delivery as a clinical benefit, however, E3 might be a weak activator of longevity, in contrast to E1 and E2. ${ }^{25}$ The anti-aging effect of $17 ß-E 2$ is clear from lower lipid peroxidation and liver dysfunction parameters, as well as histological observation, but the mechanism of estrogen action on SIRT1 expression is unclear. ${ }^{26}$ The effects of estrogen and bisphenol A on expression of histone-modifying enzymes vary, and estrogen receptor and AR may both be important in therapeutic intervention. ${ }^{27}$ SIRT1 activities have also been linked to estrogenic effects through interaction with the G-protein coupled membrane bound estrogen receptor. ${ }^{28}$

There is widespread interest in sex hormones because of their apparent anti-aging effects. The Vitex agnus-castus fruit improves features of aging, such as oxidative stress, female sex hormone deficiency, and an atrophic endometrium. ${ }^{29}$ In general, anti-aging effects are associated with reducing oxidative stress and supplementation of female sex hormones. Thus, antioxidants (vitamin E, vitamin $\mathrm{C}$, and carotenoids) and hormones (growth hormone, testosterone, DHEA, and vitamin D) can serve as anti-aging therapies. ${ }^{30}$ The present study focused on the bioactivities of sex hormones in anti-aging. A further study is needed to examine involvement of antioxidants on anti-aging in HAECs.

We found that high glucose had a significant suppressive effect on basal- and DHEA-induced SIRT1 expression in HAECs. We have also observed a similar phenomenon of inhibition of SIRT1 expression by high glucose in human monocyte leukemia cells, THP1 cells. ${ }^{3}$ High glucose may impair ER $\alpha$ transcriptional activity by inhibiting $\beta$-catenin signaling in osteoblastic MC3T3-E1 cells, leading to decreased bone formation in a ligand-dependent or -independent manner. ${ }^{31}$ Testosterone prevents pancreatic ß-cell apoptosis due to glucotoxicity through reduction of expression of ATGR1 and its signaling pathway. ${ }^{32}$ In the present study, testosterone-induced SIRT1 expression was not 
inhibited by high glucose. Paradoxically, we observed augmentation of E2-induced SIRT1 expression by high glucose. Responsiveness to $17 \AA-E 2$ in primary human osteoblasts is modulated differentially by a high glucose concentration, which supports our results. Androgen replacement therapy could be effective for type 2 diabetes-induced ED and is a potential ED treatment,but we found that high glucose significantly inhibited DHEA-induced SIRT1 expression. Therefore, based on these previous reports and our results, androgen replacement therapy for T2DM-induced ED might be weakened by poor control of blood glucose..$^{33,34}$

Activation of SIRT 1 by resveratrolameliorated cardiac injuries in a mouse model of dilated cardiomyopathy (DCM) through PGC-1 $\alpha$-mediated mitochondrial regulation, and SIRT activation by sex hormones may be a therapeutic target for DCM. ${ }^{35}$ Control of oxidative stress by AMPK activation or antioxidants could restore normal estrogen responses, even in hyperglycemia. ${ }^{14}$ Consistent with this, we found that E1- and E3-induced SIRT1 expression was not diminished by high glucose. Testosterone regulates $\beta$-cell mass, at least in part, by $A R$ activation in $B$-cells of male rats and the $B$-cell AR is degraded under hyperglycemic conditions. ${ }^{13}$ Therefore, although testosterone-induced SIRT1 expression was not weakened under hyperglycemic conditions in vitro in the present study, hormone-induced SIRT1 expression might diminish in hyperglycemia in vivo. In contrast to high glucose, 2-deoxy-d-glucose (2-DG) extends the lifespan of $\mathrm{Hs} 68$ cells by increasing $\mathrm{NAD}^{+}$ levels and SIRT1 activity, and 2-DG has potential as a caloric restriction mimetic,therefore, 2-DG may activate SIRT1 expression in HAECs.

Study limitations. Although we applied 5 to $40 \mu \mathrm{g} / \mathrm{ml}$ as the range of each agent's concentration because of being worried about cell apoptosis due to drug toxicity, the range should be more wide to verify precisely those drugs' effect. Furthermore, the present data were represented in RT-PCR analysis using each 3 wells in 2 separated experiments, respectively; however, the study should be performed using 4 wells in 3 separate experiments to avoid the variation of reappearance.

In conclusion, our results suggest that DHEA, androstenedione, testosterone, E1, and E2 activate SIRT1 expression in HAECs, and that high glucose can inhibit basal SIRT expression, but not that induced by androgens and estrogens. Future research is required to investigate whether other hormones besides sex hormones could activate SIRT1 gene expression in HAECs.
Acknowledgment. We are grateful to Prof. Daisuke Koya, Department of Diabetes and Endocrinology, Kanazawa Medical University, Ishikawa, Japan for his academic and technical advice. We also would like to thank PALABRA Inc. for English language editing.

\section{References}

1. Diamanti-Kandarakis E, Dattilo M, Macut D, Duntas L, Gonos ES, Goulis DG, et al. Mechanisms in endocrinology: Aging and aniti-aging: a combo-endocrinology overview. Eur J Endocrinol 2017; 176: R283-R308.

2. Moore RL, Dai Y, Faller DV. Sirtuin 1 (SIRT1) and steroid hormone receptor activity in cancer. J Endocrinol 2012; 213: 37-48.

3. Tsuchiya T, Endo A, Tsujikado K, Inukai T. Involvement of resveratrol and $\omega-3$ polyunsaturated fatty acids on sirtuin 1 gene expression in THP1 cells. Am J Med Sci 2017; 354: 415-422.

4. Chen CY, Wu CC, Huang YC, Hung CF, Wang LJ. Gender differences in the relationships among neurosteroid serum levels, cognitive function, and quality of life. Neuropsychiatr Dis Treat 2018; 14: 2389-2399.

5. Fu M, Liu M, Sauve AA, Jiao X, Zhang X, Wu X, et al. Hormonal control of androgen receptor function through SIRT1. Mol Cell Biol 2006; 26: 8122-8135.

6. Yang NC, Song TY, Chen MY, Hu ML. Effects of 2-deoxyglucose and dehydroepiandrosterone on intracellular $\mathrm{NAD}(+)$ level, SIRT1 activity and replicative lifespan of human Hs68 cells. Biogerontology 2011; 12: 527-536.

7. Cen J, Zhang H, Liu Y, Deng M, Tang S, Liu W, et al. Anti-aging effect of estrogen on telomerase activity in ovariectomised rats-animal model for menopause. Gynecol Endocrinol 2015; 31: 582-585.

8. Vasconsuelo A, Milanesi L, Boland R. Actions of 17ß-estradiol and testosterone in the mitochondria and their implications in aging. Ageing Res Rev 2013; 12: 907-917.

9. Nawata H, Yanase T, Goto K, Okabe T, Ashida K. Mechanism of action of anti-aging DHEA-S and the replacement of DHEA-S. Mech Ageing Dev 2002; 123: 1101-1106.

10. Lee CH, Su SC, Chiang CF, Chien CY, Hsu CC, Yu TY, et al. Estrogen modulates vascular smooth muscle cell function through downregulation of SIRT1. Oncotarget 2017; 8: 110039-110051.

11. Kumar P, Taha A, Kale RK, Cowsik SM, Baquer NZ. Physiological and biochemical effects of 17 ßestradiol in aging female rat brain. Exp Gerontol 2011; 46: 597-605.

12. Pinton G, Nilsson S, Moro L. Targeting estrogen receptor beta (ERß) for treatment of ovarian cancer: importance of KDM6B and SIRT1 for ERß expression and functionality. Oncogenesis 2018; 7: 15 .

13. Harada N, Yoda Y, Yotsumoto Y, Masuda T, Takahashi Y, Katsuki T, et al. Androgen signalingß expands $ß$-cell mass in male rats and $B$-cell androgen receptor is degraded under highglucose conditions. Am J Physiol Endocrinol Metab 2018; 314 : E274-E286.

14. Chakrabarti S, Davidge ST. High glucose-induced oxidative stress alters estrogen effects on ERalpha and ERbeta in human endothelial cells: reversal by AMPK activator. J Steroid Biochem Mol Biol 2009; 117: 99-106.

15. Wu Y, Zhang Q, Zhang R. Kaempferol targets estrogen-related receptor $\alpha$ and suppresses the angiogenesis of human retinal endothelial cells under high glucose conditions. Exp Ther Med 2017; 14: 5576-5582. 
16. Tennant JR. Evaluation of the trypan blue technique for determination of cell viability. Transplantation 1964; 2: 685-694.

17. Samaras N, Papadopoulou MA, Samaras D, Ongaro F. Off-label use of hormones as an antiaging strategy: a review. Clin Interv Aging 2014; 9: 1175-1186.

18. Ota H, Akishita M, Akiyoshi T, Kahyo T, Setou M, Sumito O, et al. Testosterone deficiency accelerates neuronal and vascular aging of SAMP8 mice: protective role of eNOS and SIRT1. PLos ONE 2012; 7: e29598.

19. Liu L, Wang D, Li L, Ding X, Ma H. Dehydroepiandrosterone inhibits cell proliferation and improves viability by regulating $S$ phase and mitochondrial permeability in primary rat Leydig cells. Mol Med Rep 2016; 14: 705-714.

20. Lee MJ, Kim EH, Lee SA, Kang YM, Jung CH, Yoon $\mathrm{HK}$, et al. Dehydroepiandrosterone prevents linoleic acidinduced endothelial cell senescence by increasing autopthagy. Metabolism 2015; 64: 1134-1145.

21. Sprando RL, Collins TF, Black TN, Orenjnik N, Grundel E, Ruggles DI. Effects of androstenedione on in utero development in rats. Food Chem Toxicol 2004; 42: 917-924.

22. Hsu SC, Huang SM, Lin SH, Ka SM, Chen A, Shin MF, et al. Testosterone increases renal anti-aging klotho gene expresiion via the androgen receptor-mediated pathway. Biochem J 2014; 464: 221-229.

23. Garratt M, Lagerborg KA, Tsai YM, Galecki A, Jain M, Miller RA. Male lifespan extension with $17-\alpha$ estradiol is linked to a sex-specific metabolomics response modulated by gonadal hormones in mice. Aging Cell 2018; 27: e12786.

24. Guo JM, Shu H, Wang L, Xu JJ, Niu XC, Zhang L. SIRT1dependent AMPK pathway on the protection of estrogen against ischemic brain injury. CNS Neurosci Ther 2017; 23: 360-369.

25. Ali ES, Mangold C, Peiris AN. Estriol: emerging clinical benefits. Menopause 2017; 24: 1081-1085.

26. Hamden K, Carreau S, Ellouz F, Masmoudi H, El FA. Protective effect of 17 beta-estradiol on oxidative stress and liver dysfunction in aged male rats. J Physiol Biochem 2007; 63: 195-201.
27. Burton K, Shaw L, Morey LM. Differential effect of estradiol and bisphenol A on Set 8 and Sirt 1 expression in prostate cancer. Toxicol Reo 2015; 2: 817-823.

28. Liarte S, Alonso-Romero JL, Nicolas FJ. SIRT1 signaling cooperation for breast cancer onset and progression. Front Endocrinol (Lausanne) 2018; 9: 552.

29. Ahangarpour A, Najimi SA, Farbood Y. Effects of Vitex agnuscastus fruit on sex hormones and antioxidant indices in a d-galactose-induced aging female mouse model. J Chin Med Assoc 2016; 79: 589-596.

30. Kamel NS, Gammack J, Cepeda O, Flaherty JH. Antioxidants and hormones as antiaging therapies: high hopes, disappointing results. Cleve Clin J Med 2006; 73: 1049-1056.

31. Wang R, Gao D, Zhou Y, Cehn L, Luo B, Yu Y, et al. High glucose impaired estrogen receptor alpha signaling via $B$-catenin in osteoblastic MC3T3-E1. J Steroid Biochem Mol Biol 2017; 174: 276-283.

32. Kooptiwut S, Hanchang W, Semprasert N, Junking M, Limjindaporn T, Yenchitsomanus PT, et al. Testosterone reduces AGTR1 expression to prevent $ß$-cell and islet apoptosis from glucotoxicity. J Endocrinol 2015; 224: 215-224.

33. Somjen D, Katzburg S, Kohen F, Gayer B, Sharon O, Hendel D, et al. Responsiveness to estradiol-17beta and to phytoestrogens in primary human osteoblasts is modulated differentially by high glucose concentration. J Steroid Biochem Mol Biol 2006; 99: 139-146.

34. Kataoka T, Hotta Y, Maeda Y, Kimura K. Assessment of androgen replacement therapy for erectile function in rats with type 2 diabetes mellitus by examining nitric oxide-related and inflammatory factors. J Sex Med 2014; 11: 920-929,

35. Ma S, Feng J, Zhang R, Chen J, Han D, Li X, et al. SIRT1 activation by resveratrol alleviates cardiac dysfunction via mitochondrial regulation in diabetic cardiomyopathy mice. Oxid Med Cell Longev 2017; 2017: 4602715. 